\title{
Statistical Mechanics of Neocortical Interactions. EEG Dispersion Relations
}

\section{LESTER INGBER}

Abstract-An approach is explicitly formulated to blend a local with a global theory to investigate oscillatory neocortical firings, to determine the source and the informationprocessing nature of the alpha rhythm. The basis of this optimism is founded on a statistical mechanical theory of neocortical interactions which has had success in numerically detailing properties of short-term-memory (STM) capacity at the mesoscopic scales of columnar interactions, and which is consistent with other theory deriving similar dispersion relations at the macroscopic scales of electroencephalographic (EEG) and magnetoencephalographic (MEG) activity.

Manuscript received 13 March 1984. This project has been supported entirely by personal contributions to Physical Studies Institute and to the University of California at San Diego Physical Studies Institute agency account through the Institute for Pure and Applied Physical Sciences.

The author is with Physical Studies Institute, Drawer W, Solana Beach, California 92075, and the Institute for Pure and Applied Physical Sciences, University of California at San Diego, La Jolla, California 92093. 


\section{OBJECTIVES}

If the development of artificial intelligence systems is to benefit from knowledge of how neocortex processes macroscopic patterned information at multiple spatial-temporal scales, then this knowledge must be gained by at least testing viable theoretical formulations based on neocortical properties against empirical data plausibly related to such processing. Towards this end, in addition to the neuroscientific relevance of this work, an approach is formulated to determine just what proportion of local and global cortical circuitry gives rise to the alpha frequency. This has strong implications for other behavioral studies which seek correlations between macroscopic EEG-MEG data and their underlying neuronal mechanisms. This calculation is an essential bridge to understand how neuronal specificity provides mechanisms underlying neuropsychological states, e.g., selective and global "attention." The statistical mechanical techniques employed are quite general [1]. E.g., they have been applied to study nucleonnucleon velocity-dependent [2] Riemannian contributions to the binding energy of nuclear matter [3,4], and to study the nonlinear dynamics of financial markets [5]. The former application of these mathematical techniques yields insights into representing mesoscopic firing patterns by eigenfunctions of a Lagrangian; the latter application is particularly interesting in the context of describing neocortical interactions more as a "neural throng" reminiscent of social interactions [6], than as "hard-wired" simple local circuits.

\section{BACKGROUND}

Statistical Mechanics of Neocortical Interactions. A series of published studies, have demonstrated that several scales of neocortical interactions can be consistently analyzed with the use of methods of modern nonlinear nonequilibrium statistical mechanics [7-10]. A more extensive background for these studies, with a fairly comprehensive set of references to other approaches, is detailed in these papers, but Appendix A gives an outline of these calculations. The formation, stability, and interaction of spatialtemporal patterns of columnar firings now can be explicitly calculated, to test hypothesized mechanisms 
relating to information processing. A detailed scenario has been calculated of columnar coding of external stimuli, short-term storage via hysteresis, and long-term storage via synaptic modification [8]. This development supports the possibility of parallel processing of local information via microscopic circuits and of global patterned information via mesoscopic columnar mechanisms [11-13].

One of the most dramatic successes of this theory has been to produce a nonphenomenological calculation of a macroscopic "observable" from microscopic synaptic dynamics: the derivation of STM capacity $[9,10]$, i.e., the "rule of $7 \pm 2$." [14-16] This theory demonstrates that empirical values of chemical and electrical parameters of synaptic interactions establish several minima of the path-integral Lagrangian as a function of excitatory and inhibitory columnar firings. The number of possible minima, their time scales of hysteresis and probable reverberations, and their nearest-neighbor (NN) columnar interactions are all consistent with well-established empirical rules of human STM capacity. Both the nonlinear and statistical natures of the interactions developed by this theory are tested by the derivation of STM capacity. Thus, aspects of conscious experience are derived from neuronal firing patterns, using modern methods of nonlinear nonequilibrium statistical mechanics to develop realistic explicit synaptic interactions. This result at least partially justifies the process by which microscopic activity has been statistically developed to describe mesoscopic and macroscopic activity. For example, although future refinements can take into consideration state-dependent complex interactions among intra-neuronal components $[17,18]$, the assumptions outlined in Appendix A appear to suffice to detail STM capacity.

In the wake of this interesting result obtained for STM capacity, this paper speculates that this theory also be applied to macroscopic EEG-MEG phenomena. As outlined in Appendix A, microscopic neuronal synaptic interactions, consistent with anatomical observations, are first spatially averaged over minicolumnar afferent and macrocolumnar efferent domains, defining a physiological "mesocolumn." These spatially ordered domains, $\sim 10^{-2} \mathrm{~cm}$, retain intimate contact with the original physical synaptic parameters, are consistent with observed columnar physiology, and are a suitable substrate for macroscopic spatial-temporal regions, $\sim$ tens of $\mathrm{cm}$, described by a path-integral Lagrangian formalism of coupled excitatory-inhibitory spatial-temporal firing states. NN interactions among mesocolumns support regions of alternating columnar structures. Long-ranged influences from extrinsic and inter-regional 
afferents drive these short-ranged interactions, giving rise to columnar mechanisms affecting macroscopic activity. Within neighborhoods of established most-probable stationary firing minima determined by the Euler-Lagrange equations, the linearized field equations give rise to a dispersion relation relating firing frequencies and spatial wave vectors, i.e., exhibiting properties of classical wave phenomena.

Origins of Time Dependencies of Scalp EEG. Other researchers have developed quite different approaches to investigating macroscopic neocortical activity, e.g., stressing that systematics of alpha rhythm of EEG can be modeled by resonant modes of macroscopic dipole-layered firing patterns of neocortex [19-22]. These resonances, in linearized coupled excitatory-inhibitory spatial-temporal integral equations describing dipole-layered sources, give rise to a macroscopic dispersion relation relating firing frequencies to spatial wave vectors, consistent with empirical observations. As demonstrated in Appendix A, typical synaptic parameters result in mesoscopic dispersion relations consistent with these macroscopic dispersion relations.

While many other investigators also accept dipole layers to model EEG activity, at least to the extent of recognizing activity perpendicular to laminae, they also demonstrate that there are respectable candidates for mechanisms that might fundamentally be responsible for macroscopic activity, other than those proposed here which detail synaptic dynamics of mesocolumnar interactions [23-30]. For example, given the present lack of empirical knowledge, it is possible to formulate macroscopic neocortical activity in terms of statistics of either membrane or synaptic microscopic neuronal activities, albeit that these two are obviously empirically dependent on each other [31]. Therefore, the results derived in Appendix A might be interpreted either as suggesting that mesocolumnar activity instigates macroscopic activity, or rather as suggesting that mesocolumnar activity strongly interacts with ongoing macroscopic activity which is instigated or sustained by other mechanisms.

\section{PRESENT ISSUES}

The two approaches outlined above in Section II, i.e., local mesocolumnar versus global nonmesocolumnar, give rise to important alternative conjectures: (1) Is the alpha rhythm a global resonance 
of primarily long-ranged cortical interactions? If so, can relatively short-ranged local firing patterns effectively modulate this frequency and its harmonics, to enhance their information processing across macroscopic regions? (2) Or, does global circuitry imply boundary conditions on collective mesoscopic states of local firing patterns, and is the alpha rhythm a manifestation of these collective local firings? (3) Or, is the truth some combination of (1) and (2) above?

Using this mesocolumnar approach, within their empirical ranges, sets of synaptic parameters can be examined to determine local dispersion relations [8] similar to those obtained from the global dispersion relations [19]. The results of such a calculation, outlined in Appendix A, clearly demonstrate that this local theory predicts alpha frequencies and spatial wave numbers compatible with those predicted by the global resonance model. For example, the possibility of generating alpha rhythm from multiple mechanisms at multiple scales of interactions, e.g., as discussed above, may account for its presence under many physiological conditions [19]. Note that these results, similar to results derived for STM capacity $[9,10]$, are not obtained by "fitting" theoretical parameters mocking neuronal mechanisms to empirical data. Rather, these results are obtained by taking reasonable synaptic parameters, developing the statistical mechanics of neocortical interactions, and then discovering that indeed they are consistent with the empirical macroscopic data. Furthermore, this theory allows the local and global approaches to complement each other at a common level of formal analysis — i.e., the "equations of motion" analogous to $\sum$ (forces $)=d($ momentum $) / d t$ describing mechanical systems: A more detailed calculation will include contributions from most probable states of the stochastically averaged microscopic system in the local approach, i.e., the linearized Euler-Lagrange equations, and will include contributions from normal modes of the linearized macroscopic system in the global approach, i.e., resonances of the dipole field equations.

It is plausible that studies of the source of the alpha rhythm will give direct insight into related mechanisms underlying evoked potentials. In any case, initially a study the Euler-Lagrange variational equations can determine just what kinds of spatial-temporal structures can be supported by the mesocolumnar system, given initial driving forces that match/mismatch firing eigenfunctions (patterns of columnar firings) currently possessed by a given set of synaptic parameters, and under conditions of 
plastically changing synaptic parameters reflecting changes of bases of eigenfunctions. However, in contrast to the alpha rhythm being a gauge of a general alertness to process information, the time-locked averaged evoked potentials appear to be a gauge of more selective attention to information being processed. Therefore, to derive the nature of evoked potentials, it is more likely that more details of local interaction among columnar interactions must be included, properly short of neuronal specificity of the specific information being processed to not refute the proper level of generality of these events. Laminar circuitry can be included in the statistical mechanics paradigm developed [8]. Therefore, ultimately Monte Carlo importance sampling techniques are to be extended to find the response of neocortex several hundred milliseconds after an initial excitation of $\sim 100$ milliseconds duration, e.g., analogous to thalamocortical stimulation. Stability analyses must be made of these solutions in the context of the original nonlinear equations. Also laminar circuitry is to be included in both the local and global models. Previous papers have detailed how this can be realized, but more numerical study is needed to determine the degree to which this can be accomplished. The solutions will be tested by their goodness-of-fit to existing EEG data normalized to flat space [19,21].

\section{APPENDIX A}

Microscopic Neurons. The microscopic probability $p_{\sigma_{j}}$ for neuron $j$ firing $\left(\sigma_{j}=+1\right.$ if $j$ fires, $\sigma_{j}=-1$ if it does not) is derived from folding a process $\Psi$ for the distribution of $q$ chemical quanta transmitted across a synaptic cleft, with a Gaussian process $\Gamma$ for the distribution of the net effect of postsynaptic interaction as it affects the electrical activity at the axonal trigger zone. Each quanta contains thousands of molecules of neurotransmitter. The probability $p_{\sigma_{j}}$ is essentially the same for $\Psi$ taken to be a Poisson or a Gaussian distribution [7]. For $\Psi$ Poisson, the mean efficacy is given as

$$
a_{j k}^{*}=\frac{1}{2} A_{j k}^{*}\left(\sigma_{k}+1\right)+B_{j k}^{*}
$$

where $A_{j k}^{*}$ is the activity induced if the presynaptic neuron $k$ fires, and $B_{j k}^{*}$ is a spontaneous background. $A_{j k}^{*}$ and $B_{j k}^{*}$ are on the order of $0.001-0.01$, and for a "typical" neuron there may be as many as 
$N^{*} \sim 10^{4}-10^{5}$ presynaptic neurons, most emanating locally from within the the range of a macrocolumn of spatial extent $\sim 1 \mathrm{~mm}$. The distribution $\Gamma$ has mean $q v_{j k}$ and variance $\sqrt{q} \phi_{j k}$, where $v_{j k}$ and $\phi_{j k}$ are the net electrical potential and its variance, resp., at the trigger zone; $\left|v_{j k}\right|$ and $\phi_{j k}$ are $\sim 0.1 \mathrm{mV}$, where $v_{j k}$ is positive for excitatory interactions and is negative for inhibitory interactions. Neuron $j$ most likely fires if the threshold potential $V_{j}$ is exceeded within a neuronal relaxation time of $\tau_{n} \sim 5-10$ msec. $p_{\sigma_{j}}$ is derived to be

$$
\begin{aligned}
& p_{\sigma_{j}} \approx \frac{\exp \left(-\sigma_{j} F_{j}\right)}{\exp F_{j}+\exp -F_{j}}, \\
& F_{j}=\frac{\left(V_{j}-\sum_{k} a_{j k}^{*} v_{j k}\right)}{\left(\pi \sum_{k^{\prime}} a_{j k^{\prime}}^{*}\left(v_{j k^{\prime}}{ }^{2}+\phi_{j k^{\prime}}{ }^{2}\right)\right)^{1 / 2}} .
\end{aligned}
$$

Mesoscopic Domains. A mesoscopic probability distribution $P$ is developed for an afferent minicolumn of $N \sim 10^{2}$ neurons, with spatial extent $\rho \sim 10^{2} \mu \mathrm{m}$ and temporal relaxation $\tau \geq \tau_{n}$, having excitatory (E) firing $M^{E}$ and inhibitory $(I)$ firing $M^{I},-N^{G} \leq M^{G} \leq N^{G}$, where $G=E$ or $I$. $P$ is a response to efferent input within the extent of a macrocolumn of $N^{*}$ neurons. As minicolumns are sensitive to one to several neuronal afferents within $\tau_{n}$, the relaxation time $\tau$ for mesocolumns is of the same order as the relaxation time $\tau_{n}$ for neurons. $E$ and $I$ type neurons have chemically independent synaptic interactions in neocortex, although the firing of a neuron is affected by the contribution from $\bar{G}=$ $E$ and $I$ neurons. A mesocolumn is defined as this afferent minicolumn and efferent macrocolumn scaled down to minicolumnar size, expressing the convergence and divergence of neocortical interactions. NN mesocolumnar interactions are defined by overlapping efferent macrocolumnar domains, with centers offset within the extent of a minicolumn. The net effect is to average over the $j k$ neurons, yielding $G G^{\prime}$ columnar interactions. The efferent scaling of $M^{* G}$ macrocolumnar efferent firings to $M^{G}=\left(N / N^{*}\right) M^{* G}$ is conveniently done by simultaneously scaling $A_{j k}^{*} \rightarrow\left(N / N^{*}\right) A_{j k}$ and $B_{j k}^{*} \rightarrow\left(N / N^{*}\right) B_{j k}$.

$$
P=\sum_{\sigma_{j}} \delta\left(\sum_{j E} \sigma_{j}-M^{E}(r ; t+\tau)\right) \delta\left(\sum_{j I} \sigma_{j}-M^{I}(r ; t+\tau)\right) \prod_{j}^{N} p_{\sigma_{j}}
$$




$$
\begin{gathered}
\approx \prod_{G}\left(2 \pi \tau g^{G G}\right)^{-1 / 2} \exp \left(-N \tau \underline{L}^{G}\right), \\
\underline{L}^{G}=\left(\dot{M}^{G}-g^{G}\right)^{2} /\left(2 N g^{G G}\right)+M^{G} J_{G} /(2 N \tau)-\underline{V}^{\prime G}, \\
\underline{V}^{\prime G} \rightarrow \sum_{G^{\prime}}{\underline{V^{\prime \prime}}}_{G^{\prime}}^{G}\left(\rho \nabla M^{G^{\prime}}\right)^{2}, \\
g^{G}=-\tau^{-1}\left(M^{G}+N^{G} \tanh F^{G}\right), g^{G G}=\tau^{-1} N^{G} \operatorname{sech}^{2} F^{G}, \\
F^{G}=\frac{\left[V^{G}-\sum_{G^{\prime}} a_{G^{\prime}}^{G} v_{G^{\prime}}^{G} N^{G^{\prime}}-\sum_{G^{\prime}} \frac{1}{2} A_{G^{\prime}}^{G} v_{G^{\prime}}^{G} M^{G^{\prime}}\right]}{\left\{\pi \sum_{G^{\prime}}\left[\left(v_{G^{\prime}}^{G}\right)^{2}+\left(\phi_{G^{\prime}}^{G}\right)^{2}\right]\left(a_{G^{\prime}}^{G} N^{G^{\prime}}+\frac{1}{2} A_{G^{\prime}}^{G} M^{G^{\prime}}\right)\right\}^{1 / 2}},
\end{gathered}
$$

where $a_{G^{\prime}}^{G}=\frac{1}{2} A_{G^{\prime}}^{G}+B_{G^{\prime}}^{G}$, and the NN differential interactions $\underline{V}_{G^{\prime}}^{\prime G}$ are further specified in other papers [9]. The $J_{G}$ are constraints on $M^{G}$ from long-ranged fibers, e.g., from thalamocortical cortical, ipsilateral association and contralateral commissural excitatory fibers extrinsic to macrocolumns.

Macroscopic Regions. The macroscopic probability $\tilde{P}\left[\tilde{M}(t) \mid \tilde{M}\left(t_{0}\right)\right]$ is developed by folding $\Lambda \sim 5 \times 10^{5}$ mesocolumns of spatial extent $\Omega \sim 5 \times 10^{9} \mu \mathrm{m}^{2}$, labeled by $v(v=\Lambda+1 \equiv 1)$, and folding the differential propagator $P^{\theta}$ for $u+1$ time periods, spanning time $t-t_{0}=s \theta$, each period of duration $\theta \leq \tau$ labeled by $s$. Boundary conditions on the macroscopic regions are defined to be $\tilde{M}_{u+1}=\tilde{M}(t)$ and $\tilde{M}_{0}=\tilde{M}\left(t_{0}\right), \tilde{M}=\left\{M^{G v}\right\}$, and the Einstein convention of summing over factors with repeated $G$ indices is henceforth assumed, except when vertical bars appear on an index, e.g., $|G|$. The prepoint discretization of $\underline{L}(M), \theta \dot{M}^{G}\left(t^{\prime}\right) \rightarrow M_{s+1}^{G}-M_{s}^{G}$ and $M^{G}\left(t^{\prime}\right) \rightarrow M_{s}^{G}$, is derived from the biophysics of neocortex. This is not equivalent to the Stratonovich midpoint discretization of a proper Feynman Lagrangian $\underline{L}_{F}, \theta \dot{M}^{G}\left(t^{\prime}\right) \rightarrow M_{s+1}^{G}-M_{s}^{G}$ and $M^{G}\left(t^{\prime}\right) \rightarrow \frac{1}{2}\left(M_{s+1}^{G}+M_{s}^{G}\right)$ [1]. The discretization and the Lagrangian must be defined consistently to give an invariant scalar $g^{1 / 2} P(M)$, where $g=\left\|g_{G G^{\prime}}\right\|$, $g_{G G^{\prime}}=\left(g^{G G^{\prime}}\right)^{-1}$. The covariant Feynman Lagrangian is defined in terms of a stationary principle, and the 
transformation to the Stratonovich discretization permits the use of the standard calculus.

$$
\begin{aligned}
& \tilde{P}\left[\tilde{M} \mid \tilde{M}_{0}\right]=\int \cdots \int \underline{D} \tilde{M} \exp \left(-N \tilde{S}_{F}\right) \delta[\tilde{M}=\tilde{M}(t)] \delta\left[\tilde{M}_{0}=\tilde{M}\left(t_{0}\right)\right] \\
& \tilde{S}_{F}=\min \Lambda \Omega^{-1} \int d t^{\prime} \int d^{2} r \underline{L}_{F}, \\
& \underline{D} \tilde{M}=\prod_{v=1}^{\Lambda} \prod_{G}^{E, I} \prod_{s=1}^{u+1}\left[(2 \pi \theta)^{-1 / 2}\left(g_{s}^{v}\right)^{1 / 4}\right] \prod_{s^{\prime}=1}^{u} d M_{s^{\prime}}^{G v}, \\
& \underline{L} F=\frac{1}{2} N^{-1}\left(\dot{M}^{G}-h^{G}\right) g_{G G^{\prime}}\left(\dot{M}^{G^{\prime}}-h^{G^{\prime}}\right)-\underline{V}, \\
& h^{G}=g^{G}-\frac{1}{2} g^{-1 / 2}\left(g^{1 / 2} g^{G G^{\prime}}\right)_{G^{\prime}}, h_{; G}^{G}=g^{-1 / 2}\left(g^{1 / 2} h^{G}\right)_{, G}, \\
& \underline{V}=\underline{V}^{\prime}-\left(\frac{1}{2} h_{; G}^{G}+R / 6\right) / N, \underline{V}^{\prime}=\underline{V}^{E}+\underline{V}^{\prime I}-M^{G} J_{G} /(2 N \tau),
\end{aligned}
$$

where $[\cdots]_{, G}$ denotes $\partial[\cdots] / \partial M^{G}$, and “min" specifies that the short-time propagator is evaluated by expanding about that path which makes the action $\tilde{S}_{F}$ stationary. The Riemannian curvature $R$ arises from the nonlinear inverse variance $g_{G G^{\prime}}$, which is a bona fide metric of this parameter space,

$$
R=g^{-1}\left(g_{E E, I I}+g_{I I, E E}\right)-\frac{1}{2} g^{-2}\left[g_{I I}\left(g_{E E, E} g_{I I, E}+g_{E E, I}^{2}\right)+g_{E E}\left(g_{I I, I} g_{E E, I}+g_{I I, E}^{2}\right)\right]
$$

This path-integral representation is equivalent to the Fokker-Planck and Langevin differential-equation representations $[1,9]$.

Euler-Lagange Variational Equations. The Euler-Lagrange variational equations associated with $\underline{L}_{F}$ leads to a set of 12 coupled first-order differential equations, with coefficients nonlinear in $M^{G}$, in the 12 variables $\left\{M^{G}, \dot{M}^{G}, \ddot{M}^{G}, \nabla M^{G}, \nabla^{2} M^{G}\right\}$ in $(r ; t)$ space. In the neighborhood of extrema $\ll \bar{M}^{G} \gg$, $\underline{L}_{F}$ can be expanded as a Ginzburg-Landau polynomial. To investigate first-order linear oscillatory states, 
only powers up to 2 in each variable are kept, and from this the variational principle leads to a relatively simple set of coupled linear differential equations with constant coefficients:

$$
\begin{aligned}
& 0=\hat{\delta} \underline{L}_{F}=\underline{L}_{F, \dot{G}: t}-\hat{\delta}_{G} \underline{L}_{F} \\
& \left.\approx-\underline{f}_{|G|} \ddot{M}^{|G|}+\underline{f}_{G}^{1} \underline{\dot{M}}^{G\urcorner}-\underline{g}_{|G|} \nabla^{2} \underline{M}^{|G|}+\underline{b}_{|G|} \underline{M}^{|G|}+\underline{b}^{M} \underline{M}^{G\urcorner}, G\right\urcorner \neq G, \\
& {[\cdots]_{, \dot{G}: t}=[\cdots]_{, \dot{G} G^{\prime}} \dot{M}^{G^{\prime}}+[\cdots]_{, \dot{G} \dot{G}^{\prime}} \ddot{M}^{G^{\prime}},} \\
& \underline{M}^{G}=M^{G}-\ll \bar{M}^{G} \gg, \underline{f}_{E}^{1}=-\underline{f}_{-}^{1} \equiv \underline{f} .
\end{aligned}
$$

These equations are then Fourier transformed and the resulting dispersion relation is examined to determine for which values of the synaptic parameters and of $\underline{\xi}$, the conjugate variable to $r$, can oscillatory states, $\omega(\underline{\xi})$, persist. E.g., solutions are sought of the form

$$
\begin{aligned}
& \underline{M}^{G}=\operatorname{Re} \underline{M}_{\mathrm{osc}}^{G} \exp [-i(\underline{\xi} \cdot r-\omega t)], \\
& \underline{M}_{\mathrm{osc}}^{G}(r, t)=\int d^{2} \underline{\xi} d \omega \underline{\hat{M}}_{\mathrm{osc}}^{G}(\underline{\xi}, \omega) \exp [i(\underline{\xi} \cdot r-\omega t)] .
\end{aligned}
$$

For instance, a typical example is specified by: extrinsic sources $J_{E}=-2.63$ and $J_{I}=4.94$, $N^{E}=125, N^{I}=25, V^{G}=10 \mathrm{mV}, A^{E}=1.75, A^{I}=1.25, B^{G}=0.25$, and $v^{G}=\phi^{G}=0.1 \mathrm{mV}$. The global minima is at $\bar{M}^{E}=25$ and $\bar{M}^{I}=5$. This set of conditions yields (dispersive) dispersion relations

$$
\omega \tau= \pm\left\{-1.86+2.38(\xi \rho)^{2} ;-1.25 i+1.51 i(\xi \rho)^{2}\right\}
$$

where $\xi=|\xi|$. The propagation velocity defined by $d \omega / d \xi$ is $\sim 1 \mathrm{~cm} / \mathrm{sec}$, taking typical wave-numbers $\xi$ to correspond to macrocolumnar distances $\sim 30 \rho$. Calculated frequencies $\omega$ are on the order of EEG frequencies $\sim 10^{2} \mathrm{sec}^{-1}$. These mesoscopic propagation velocities permit processing over several minicolumns $\sim 10^{-1} \mathrm{~cm}$, simultaneous with processing of mesoscopic interactions over tens of $\mathrm{cm}$ via 
association fibers with propagation velocities $\sim 600-900 \mathrm{~cm} / \mathrm{sec}$. I.e., both can occur within $\sim 10^{-1} \mathrm{sec}$.

Note that this propagation velocity is not "slow": Visual selective attention moves at $\sim 8$ $\mathrm{msec} /$ degree [32], which is $\sim 1 / 2 \mathrm{~mm} / \mathrm{sec}$, if a macrocolumn of $\sim \mathrm{mm}^{2}$ is assumed to span 180 degrees. This suggests that $\mathrm{NN}$ interactions play some part in disengaging and orienting selective attention. 


\section{REFERENCES}

[1] F. Langouche, D. Roekaerts, and E. Tirapegui, Functional Integration and Semiclassical Expansions, Reidel, Dordrecht, The Netherlands, 1982.

[2] L. Ingber, "Nuclear forces," Phys. Rev., vol. 174, pp. 1250-1263, 1968.

[3] L. Ingber, "Riemannian corrections to velocity-dependent nuclear forces," Phys. Rev. C, vol. 28, pp. 2536-2539, 1983.

[4] L. Ingber, "Path-integral Riemannian contributions to nuclear Schrodinger equation," Phys. Rev. D, vol. 29, pp. 1171-1174, 1984.

[5] L. Ingber, "Statistical mechanics of nonlinear nonequilibrium financial markets," Math. Modelling, vol. 5, pp. 343-361, 1984.

[6] T.H. Bullock, "Identifiable and addressed neurons in the vertebrates: How far can we go?," in Neurobiology of the Mauthner Cell, , ed. by D. Faber and H. Korn, Raven, New York, 1978.

[7] L. Ingber, "Statistical mechanics of neocortical interactions. I. Basic formulation," Physica D, vol. 5, pp. 83-107, 1982.

[8] L. Ingber, "Statistical mechanics of neocortical interactions. Dynamics of synaptic modification," Phys. Rev. A, vol. 28, pp. 395-416, 1983.

[9] L. Ingber, "Statistical mechanics of neocortical interactions. Derivation of short-term-memory capacity," Phys. Rev. A, vol. 29, pp. 3346-3358, 1984.

[10] L. Ingber, "Statistical mechanics of neocortical interactions: Stability and duration of the 7+-2 rule of short-term-memory capacity," Phys. Rev. A, vol. 31, pp. 1183-1186, 1985.

[11] L. Ingber, “Editorial: Learning to learn,” Explore, vol. 7, pp. 5-8, 1972.

[12] L. Ingber, “Attention, physics and teaching,” J. Social Biol. Struct., vol. 4, pp. 225-235, 1981.

[13] L. Ingber, “Towards a unified brain theory,” J. Social Biol. Struct., vol. 4, pp. 211-224, 1981. 
[14] G.A. Miller, "The magical number seven, plus or minus two," Psychol. Rev., vol. 63, pp. 81-97, 1956.

[15] K.A. Ericsson and W.G. Chase, "Exceptional memory," Am. Sci., vol. 70, pp. 607-615, 1982.

[16] S. Grossberg, "Associative and competitive principles of learning and development," in Competition and Cooperation in Neural Nets, , ed. by S. Amari and M.A. Arbib, Springer, New York, 1982, pp. 295-341.

[17] A. Pellionisz and R. Llinás, "Tensorial approach to the geometry of brain function: cerebellar coordination via a metric tensor," Neuroscience, vol. 5, pp. 1125-1136, 1980.

[18] J.A. Wilson and C.E. Phillips, "Pre-motor non-spiking interneurons," Prog. Neurobiol., vol. 20, pp. 89-107, 1983.

[19] P.L. Nunez, Electric Fields of the Brain: The Neurophysics of EEG, Oxford University Press, London, 1981.

[20] P.L. Nunez, "A study of origins of the time dependencies of scalp EEG: I-Theoretical basis," IEEE Trans. Biomed. Eng., vol. 28, pp. 271-280, 1981.

[21] P.L. Nunez, "A study of origins of the time dependencies of scalp EEG: II—Experimental support of theory," IEEE Trans. Biomed. Eng., vol. 28, pp. 281-288, 1981.

[22] P.L. Nunez, "The brain wave equation: A model for the EEG," Math. Biosci., vol. 21, pp. 279-297, 1974.

[23] S.-I. Amari, "Field theory of self-organizing neural nets," IEEE Trans. Syst. Man Cybern., vol. 13, pp. 741-748, 1983.

[24] P. Anninos, S. Zenone, and R. Elul, “Artificial neural nets: Dependence of the EEG amplitude's probability distribution on statistical parameters," J. Theor. Biol., vol. 103, pp. 339-348, 1983.

[25] E. Basar, EEG-Brain Dynamics, Elsevier, Amsterdam, 1980. 
[26] W.J. Freeman, "Spatial properties of an EEG event in the olfactory bulb and cortex," Electroencephal. clin. Neurophysiol., vol. 44, pp. 586-605, 1978.

[27] J.G.R. Jefferys, "Initiation and spread of action potentials in granule cells maintained in vitro in slices of guinea-pig hippocampus," J. Physiol., vol. 289, pp. 375-388, 1979.

[28] W.R. Klemm and C.J. Sherry, "Serial ordering in spike trains: What's it 'trying to tell us'?," Int. J. Neurosci., vol. 14, pp. 15-33, 1981.

[29] M. Steriade, "Mechanisms underlying cortical activation: Neuronal organization and properties of the midbrain reticular core and intralaminar thalamic nuclei," in Brain Mechanisms and Perceptual Awareness, , ed. by O. Pompeiano and C.A. Marsan, Raven, New York, 1981, pp. 327-377.

[30] R.D. Traub and R. Llinás, "Hippocampal pyramidal cells: Significance of dendritic ionic conductances for neuronal function and epileptogenesis," J. Neurophysiol., vol. 42, pp. 476-496, 1979.

[31] F.E. Dudek, G.I. Hatton, and B.A. Macvicar, "Intracellular recordings from the paraventricular nucleus in slices of rat hypothalamus," J. Physiol., vol. 301, pp. 101-114, 1980.

[32] Y. Tsal, "Movements of attention across the visual field," J. Exp. Psychol., vol. 9, pp. 523-530, 1983. 